\title{
Quantification of the Impact of Ocean Acidification on Marine Calcifiers
}

By Katsunori Kimoto

Since the beginning of the Industrial Revolution, $\mathrm{CO}_{2}$ emissions into the atmosphere have been increasing continuously. The ocean has been taking up at least a quarter of the excess atmospheric $\mathrm{CO}_{2}$. The $\mathrm{CO}_{2}$ that dissolves in the ocean reacts with seawater and carbonate ions, leading to a decrease in seawater $\mathrm{pH}$, a process called ocean acidification (OA) and is often called the "second $\mathrm{CO}_{2}$ problem." OA could trigger remarkable changes in the ocean's inorganic carbon system, ultimately affecting marine ecosystems. Marine organisms that have calcium carbonate skeletons such as corals, bivalves, crustaceans, and microorganisms are especially vulnerable. It is essential to understand how OA affects biological systems in order to predict future environmental impacts on organisms and ecosystem services.

The effects of OA on marine organisms can be evaluated by morphological analysis of skeleton/shell surfaces using a light stereomicroscope and scanning electron microscopy (SEM). However, such observations only document surface features, limiting quantification of the degree of OA damage. Advances in X-ray Computed Tomography (XCT) have made it possible to obtain quantitative morphological information such as the volume, surface area, thickness, and density of target objects such as marine organism shells and skeletons. XCT can also be used to construct a precise, three-dimensional morphology with micrometer to submicrometer resolution. Very small amounts of

FIGURE 1. (left) Shelled pteropod Limacina helicina, one of the major food sources of carnivorous zooplankton, fishes, and seabirds in the polar ocean. This pteropod has a skeleton of aragonite, a form of calcium carbonate that is more soluble than calcite. (right) Threedimensional sectional microtomography image with density mapping of a planktic foraminifer, Globigerina bulloides, recovered from the western North Pacific. The colors show the denser (red) and less dense (blue) parts of the carbonate skeleton. physical damage from OA can be detected, even if the damage is inside the skeleton.

Innovative application of the latest Micro Focus XCT ( $\mu \mathrm{XCT})$ technology to low-trophic-level microzooplankton shows that the skeletal densities of shelled pteropods (sea butterflies) and planktonic foraminifera (both shown in Figure 1) are closely related to the carbonate chemistry of the surrounding seawater (e.g., Iwasaki et al., 2019; Ofstad et al., 2021). Such quantitative measures of skeletal density based on $\mu$ XCT analyses can be used to inform marine ecosystem models and improve predictions based on future $\mathrm{CO}_{2}$ emission scenarios.

In order to accelerate this research, we recently founded a service that provides quantitative information about the morphology and density of micro-sized objects based on HXCT (http://www.jamstec.go.jp/rcgc/e/mxct/) at no cost to users. The Global Ocean Acidification Observation Network (GOA-ON) has encouraged this initiative, whose overarching goal is to provide a global map of the biological impacts of OA on marine calcifiers. To achieve this goal, cooperation and collaboration among researchers in every country are necessary, and we encourage researchers to participate in this important project.

\section{REFERENCES}

Iwasaki, S., K. Kimoto, O. Sasaki, H. Kano, and H. Uchida. 2019. Sensitivity of planktic foraminiferal test bulk density to ocean acidification. Scientific Reports 9:9803, https://doi.org/10.1038/s41598-019-46041-x.

Ofstad, S., K. Zamelczyk, K. Kimoto, M. Chierici, A. Fransson, and T. Lander Rasussen. 2021. Shell density of planktonic foraminifera and pteropod species Limacina helicina in the Barents Sea: Relation to ontogeny and water chemistry. PLOS ONE, https://doi.org/10.1371/journal. pone.0249178.

ARTICLE DOI: https://doi.org/10.5670/oceanog.2021.supplement.02-19

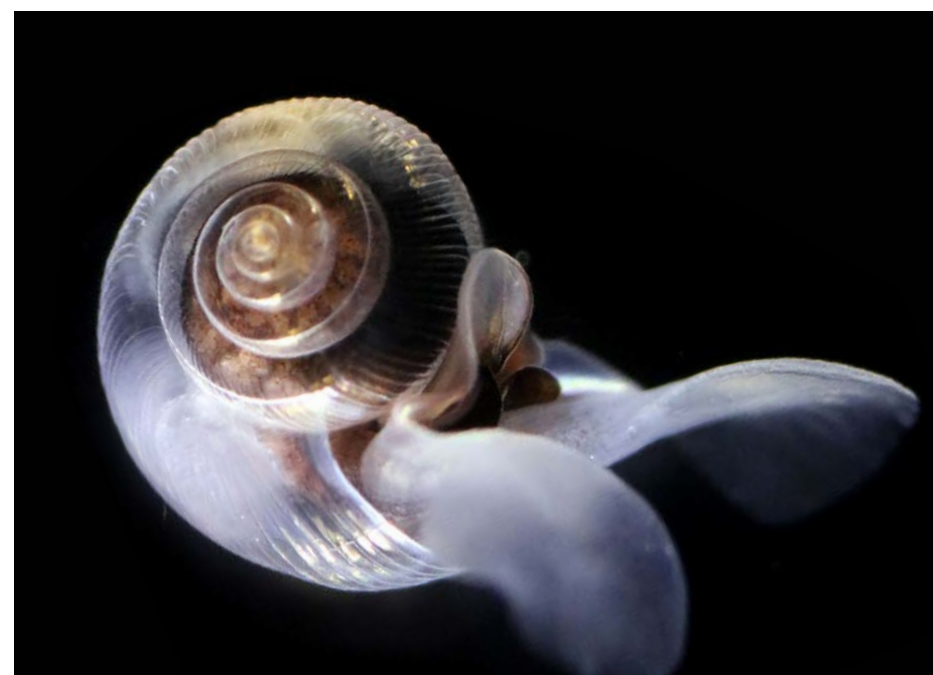




\section{AUTHORS}

Katsunori Kimoto (kimopy@jamstec.go.jp), Japan Agency for Marine-Earth Science and Technology, Japan.

\section{ARTICLE CITATION}

Kimoto, K. 2021. Quantification of the impact of ocean acidification on marine calcifiers. P. 48 in Frontiers in Ocean Observing: Documenting Ecosystems, Understanding Environmental Changes, Forecasting Hazards. E.S. Kappel, S.K. Juniper, S. Seeyave, E. Smith, and M. Visbeck, eds, A Supplement to Oceanography 34(4), https://doi.org/ 10.5670/oceanog.2021. supplement.02-19.

\section{COPYRIGHT \& USAGE}

This is an open access article made available under the terms of the Creative Commons Attribution 4.0 International License (https://creativecommons.org/ licenses/by/4.0/), which permits use, sharing, adaptation, distribution, and reproduction in any medium or format as long as users cite the materials appropriately, provide a link to the Creative Commons license, and indicate the changes that were made to the original content. 